\title{
Radiofrequency ablation of varicose veins: experience at a tertiary hospital
}

\author{
Farooq A. Ganie ${ }^{1^{*}}$, Ghulam Nabi Lone ${ }^{1}$, Mohd Yaqoob Khan ${ }^{1}$, Syed Mohsin Manzoor ${ }^{1}$, \\ Mudasir Hamid Bhat ${ }^{2}$, Syed Nisar Ahmad ${ }^{3}$ \\ ${ }^{1}$ Department of Cardiovascular and Thoracic Surgery, Sheri-I-Kashmir Institute of Medical Sciences (SKIMS), \\ Soura, Srinagar, Kashmir, India; ${ }^{2}$ Department of Radiology, Sheri-I-Kashmir Institute of Medical Sciences \\ (SKIMS), Soura, Srinagar, Kashmir, India; ${ }^{3}$ Department of Medical Oncology, Sheri-I-Kashmir Institute of \\ Medical Sciences (SKIMS), Soura, Srinagar, Kashmir, India
}

\begin{abstract}
Background and objectives: Radiofrequency ablation (RFA) is a recent modality of treatment of the affected varicose vein. In the present study, the outcome of great saphenous varicose vein disease treated by radiofrequency ablation technique was analyzed.

Methods: Patients with varicosities of the lower limb affecting mainly the great saphenous vein were (GSV) included. The procedures were carried out under spinal anesthesia. The target varicose vein was accessed by Seldinger technique and the RFA catheter advanced 2 to $3 \mathrm{~cm}$ below sapheno-femoral junction under ultrasonography (USG) guidance. A tumescent anesthetic infiltration was given in a solution of normal saline and sodium bicarbonate before the vein being ablated.

Results: The success rate of RFA was $97.5 \%$ (39 out of 40). One patient showed episodic recanalisation of vein at one year duplex colour scan. Though the complications related to procedure were negligible, one patient developed endovenous heat induced thrombosis (EHIT) and non-fatal pulmonary thromboembolism (PTE) which was managed adequately.

Conclusion: Endovenous RFA is a useful treatment modality for varicose vein disease primarily due to great saphenous insufficiency with marked symptomatic improvement and least recurrence. Although the complications are minimal, EHIT is a potential and serious complication of heat ablation.
\end{abstract}

IMC J Med Sci 2021; 15(1): 006

\section{Introduction}

Varicose veins are large, swollen veins that often appear on the legs and feet. Varicose veins are generally benign. The cause of this condition is not known precisely, however some risk factors are attributed to occupations with long standing and hormonal imbalance. The spectrum of symptoms is diverse ranging from asymptomatic engorged veins to aching pain and discomfort or skin ulceration and bleeding signaling an underlying circulatory problem. Treatment modalities involve compression stockings, specific exercises or operative procedures to remove or obliterate the affected veins. Available operative modalities include saphenous venous ligation and stripping, phlebectomy, endovenous laser therapy and radiofrequency ablation. Radiofrequency ablation is the newest of these technologies [1]. Conventional venous stripping is an invasive procedure and inflicts tremendous surgical trauma

*Correspondence: Farooq Ahmad Gganie, Department of Cardiovascular and Thoracic Surgery, Sheri-I-Kashmir Institute of Medical Sciences (SKIMS), Soura, Srinagar, Kashmir, India. Email ID: farooq.ganie@ymail.com 
to patients [2]. The blood loss is also considerable in some cases. Though laser and cryo-ablation are alternative options gaining widespread popularity but the cost consideration remain the main limiting factor for universal application in our set up. In the present study, we employed radio-frequency ablation technique to selected strata of patients with great saphenous varicose vein disease and analyzed our experience.

\section{Material and methods}

The study included patients having clinically evident varicosities of the lower limb mainly affecting the great saphenous vein (GSV). The patients were evaluated both clinically and by Doppler study. Compression ultrasonography (USG) was also done to rule out deep vein thrombosis. All the patients were admitted on day care basis with base line investigations of ECG, Chest X-ray, and a haemogram and with a fasting state of 6 hours. The procedures were carried out under spinal anesthesia. After a formal surgical drape, a standard dose of $5000 \mathrm{IU}$ of unfractionated heparin was systemically administered. The target varicose vein was accessed by Seldinger technique and the radiofrequency ablation catheter advanced 2 to 3 $\mathrm{cm}$ below sapheno-femoral junction under USG guidance. Before vein ablation - a tumescent anesthetic infiltration in a solution of normal saline and sodium bicarbonate was given all around the varicosity for added anesthesia, limited heat dissipation and tissue salvage. We used a Covidien RFA generator supporting a $100 \mathrm{~cm}$ long, 7 French catheter with a $4 \mathrm{~cm}$ spiral heating element. Each session of RFA cycle dissipates energy of 15 watts. The radiofrequency energy was delivered by serial calculated firings, with each withdrawal of $4-5 \mathrm{~cm}$ marked by a poppet on the catheter. The varicose veins were ablated just up to the knee to avoid injury to the sural nerve. The remaining below-knee varicosities were treated with injection sclerotherapy by foam. After the satisfactory ablation was achieved, the limb was stocked by an elastic crepe. An ultrasound evaluation was performed within 2 weeks of the procedure to evaluate vein occlusion, vein wall thickness, and any clot extension into the deep vein. In cases of bilateral limb disease, only the limb with severe varicosity was ablated and no attempt was made to ablate both limb varicosities in the same sitting. The patients were assessed and discharged the next morning and followed bi-weekly for two months and at one year with colour duplex scan. The study was approved by the institutional ethical board.

\section{Results}

We included 40 patients in our study. Only one limb was ablated at one time. Therefore, the results of 40 limbs of 40 patients are described here. The mean age of the patients at presentation was $41 \pm 4.24$ years with a male to female ratio of 4:1 (Table-1). The mean duration of the procedure was 37 minutes. All the 40 procedures culminated uneventfully except for one patient who developed a small subcutaneous haematoma at puncture site.

Table-1: Age and gender distribution of the study population

\begin{tabular}{llll}
\hline Parameters & Male & Female & Total \\
\hline Gender $(\mathrm{n})$ & 32 & 8 & 40 \\
Mean age $(\mathrm{yrs})$ & 38 & 44 & $41 \pm 4.24$ \\
\hline
\end{tabular}

The ablated veins were identified/palpated on table after the ablation. None of the patients developed any burn injury to surrounding tissues. One patient developed PTE due to heat induced thrombosis which was successfully thrombolised by streptokinase and discharged after few days of admission. The post procedure pain was minimal and managed by oral non opioid analgesics except for one patient who complained of persistent numbness and pain that required opioid analgesia. All the remaining 39 patients were discharged next day of procedure without any complications. All these patients resumed their usual activities on day three without the need of any medication. One patient showed episodic recanalisation of the ablated GSV on colour duplex study but had improvement in pain and heaviness. None of the patients showed any evidence of gross residual veinous reflux on follow-up duplex scans (Table-2). There was no tissue burn and gross residual veinous reflux in any of our cases. 
The success rate of thermal ablation was $97.5 \%$ (39 out of 40) with one patient showing episodic recanalisation of vein at one year duplex colour scan. Though the complications related to procedure were negligible, one patient developed EHIT and non-fatal PTE which was managed adequately.

Table-2: Complications after radiofrequency ablation $(n=40)$

\begin{tabular}{lc}
\hline Complication & Number of patients (\%) \\
\hline Haematoma & $1(2.4)$ \\
PTE due to HIT & $1(2.4)$ \\
Recanalisation (subtle) & $1(2.4)$ \\
\hline
\end{tabular}

Note: No case of tissue burn and Gross residual veinous reflux; PTE: pulmonary thromboembolism; HIT: heat induced thrombosis

\section{Discussion}

Endovascular ablation of the great saphenous vein has been proposed as a less invasive alternative to conventional ligation and stripping of varicose veins [1]. The RFA procedure involves using a catheter electrode to deliver a high-frequency alternating radio frequency current that leads to veinous spasm, collagen shrinkage and physical contraction [2]. Radiofrequency ablation is a valuable alternative to open surgery and would free up operating room time in a context of low accessibility [2]. Endovenous ablation using RFA performed as an outpatient are likely to be cost-effective treatment strategies for patients with primary unilateral GSV reflux requiring treatment [3]. Compared with conventional open surgery, RFA can be performed in the outpatient setting without the requirement for hospital admission or general anesthesia [4]. Radiofrequency ablation and foam are associated with a faster recovery and less postoperative pain than endovenous laser ablation and stripping [5].

The reported incidence of DVT is much lower in RFA (0.29-0.7\%) [6]. Minor complications of RFA include muscle cramping, localized hot flashes, induration, numbness and skin discoloration [7]. The stratified incidence of veinous thromboembolism appears to be low as reported in both randomized controlled trials and case series investigating these modalities [8]. Rarely heat induced thrombosis may occur and can have catastrophic implications. Therefore, a pre-procedural single dose of unfractionated heparin is advised. The success rate of RFA is reported high with an initial success rate of $97.7 \%$ and a significantly improved patient symptom score at 1 year [9]. The technique of endovenous thermal ablation is not only effective and safe but also a durable treatment in patients with symptomatic varicose veins [10].

Endovenous RFA is a useful treatment modality for varicose vein disease primarily due to great saphenous insufficiency with marked symptomatic improvement and least recurrence. Although the complications are minimal, EHIT is a potential and serious complication of heat ablation. Therefore, we advocate a pre-ablation dose of unfractionated heparin to all patients. The post operative recovery is quick with a high patient acceptance.

\section{References}

1. Casana R, Tolva VS, Odero A Jr, Malloggi C, Parati G. Three-year follow-up and quality of life of endovenous radiofrequency ablation of the great saphenous vein with the ClosureFast $^{\mathrm{TM}}$ procedure: Influence of $\mathrm{BMI}$ and CEAP class. Vascular. 2018 Oct; 26(5): 498-508.

2. Bergan JJ, Kumins NH, Owens EL, Sparks SR. Surgical and endovascular treatment of lower extremity venous insufficiency: J Vasc Interv Radiol. 2002 Jun; 13(6): 563-8.

3. Poder TG, Fisette JF, Bédard SK, Despatis MA. Is radio-frequency ablation of varicose veins a valuable option? A systematic review of the literature with a cost analysis. Can J Surg. 2018 Apr; 61(2): 128-138.

4. Kayssi A, Pope M, Vucemilo I, Werneck C. Endovenous radiofrequency ablation for the treatment of varicose veins: Can J Surg. 2015 Apr; 58(2): 85-86.

5. Gohel MS, Epstein DM, Davies $\mathrm{AH}$. Cost-effectiveness of traditional and endovenous treatments for varicose veins: $\mathrm{Br} \mathrm{J}$ Surg. 2010 Dec; 97(12): 1815-1823. 
6. Tamura K, Maruyama T. Mid-Term report on the safety and effectiveness of endovenous radiofrequency ablation for varicose veins. Ann Vasc Dis. 2017 Dec 25; 10(4): 398-401.

7. Izumi M, Ikeda $\mathrm{Y}$, Yamashita $\mathrm{H}$, Asaoka $\mathrm{Y}$, Fujishiro $M$, Shin $M$, Abo $Y$ : Safety and effectiveness of endovenous laser ablation combined with ligation for severe saphenous varicose veins in Japanese patients: Int Heart J. 2016; 57(1): 87-90.

8. Dermody M, Schul MW, O'Donnell TF. Thromboembolic complications of endovenous thermal ablation and foam sclerotherapy in the treatment of great saphenous vein insufficiency: Phlebology. 2015 Jun; 30(5): 357-364.
9. Jin HY, Ohe HJ, Hwang JK, Kim SD, Kim JY, Park $\mathrm{SC}$, et al. Radiofrequency ablation of varicose veins improves venous clinical severity score despite failure of complete closure of the saphenous vein after 1 year. Asian J Surg. 2017 Jan; 40(1): 48-54.

10. Ravi R, Trayler EA, Barrett DA, Diethrich EB. Endovenous thermal ablation of superficial venous insufficiency of the lower extremity: single-center experience with 3000 limbs treated in a 7-year period. J Endovasc Ther. 2009 Aug; 16(4): 500-5. 